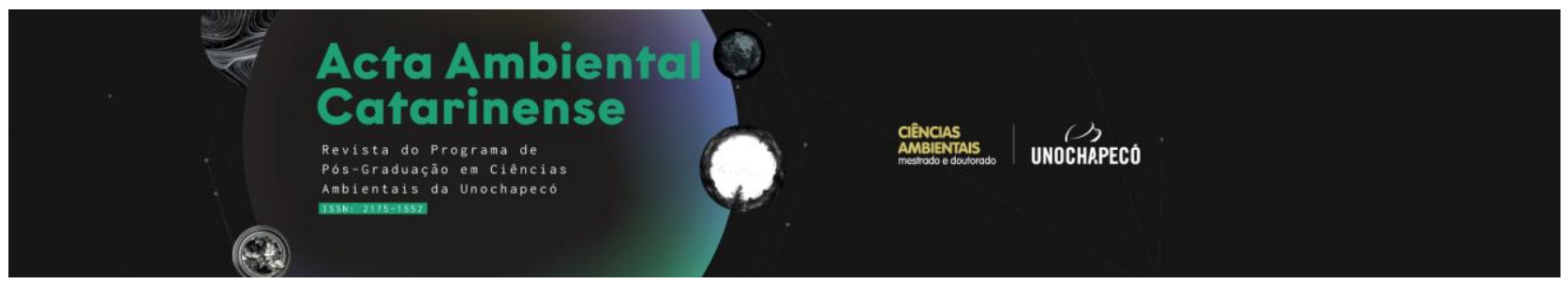

\title{
PERFIL DA PRODUÇÃO AGRÍCOLA NA REGIÃO DE SÃO CARLOS, SP: UM BALANÇO DOS ÚLTIMOS 12 ANOS
}

\author{
Bruno Salata Lima ${ }^{1}$ \\ Risely Ferraz-Almeida ${ }^{2}$
}

\begin{abstract}
Resumo
A expansão da cana-de-açúcar está mudando o perfil da produção agrícola em algumas regiões do Brasil. Com a hipótese que o aumento da área de cana-de-açúcar tem uma relação inversa com a área agrícola do milho, mas com uma relação positiva com a produção de leguminosas no sistema de sucessão cana-de-açúcar/leguminosa. O objetivo deste estudo foi monitorar o perfil da produção agrícola das culturas temporárias na região de São Carlos, São Paulo, durante 2004 a 2015. Uma meta analise foi realizada com os dados do Censo agropecuário, disponibilizado pelo Instituto Brasileiro de Geografia e Estatística. Monitorou o perfil da produção de cana-de-açúcar, amendoim, feijão, mandioca, milho, soja e tomate. Resultados mostraram que a cana-de-açúcar e o milho são as culturas temporárias com maiores expressividades na região. $\mathrm{O}$ aumento na área agrícola com cana-de-açúcar reduziu a área de produção de milho $(-0,85)$, soja $(-0,33)$, feijão $(-0,74)$ e tomate $(-$ 0,58). A importante da produção da cana-de-açúcar no cenário brasileiro é evidente para a produção de açúcar e etanol. Existe uma relação inversa da área de cana-de-açúcar com as áreas com milho e soja que é controlada pelas condições do mercado. Em contrapartida, a sucessão usando o amendoim/cana-de-açúcar promoveu uma relação harmoniosa entre as culturas com o aumento da produção de amendoim e melhoria das condições do solo, além de ser uma alternativa economicamente viável na reforma do canavial. Possivelmente, a soja também pode ser inserida nesta perspectiva nos próximos anos devido as características similares (também leguminosa) e o aumento da demanda por soja no mercado nacional e internacional.
\end{abstract}

Palavras-chave: Saccharum officinarum; Zea mays; Arachis hypogaea; Glycine max

\footnotetext{
${ }^{1}$ Mestre em Genética Evolutiva e Biologia Molecular. Rua Iwagiro Toyama, 261 - Jardim Paulistano; $13564-380$ São Carlos, SP, Brasil. E-mail: brunos_1@ hotmail.com

${ }^{2}$ Orientadora do MBA da USP/ESALQ (Universidade de São Paulo/Escola Superior de Agricultura "Luiz de Queiroz"), R. Alexandre Herculano, 120 - T6 - Vila Monteiro, Piracicaba - SP, 13418-445. E-mail: rizely@gmail.com 


\begin{abstract}
The expansion of sugarcane areas is changing the profile of agricultural production in some regions of Brazil. With the hypothesis that the increase in the area of sugarcane has an inverse relationship with the agricultural area of corn, but with a positive relationship with the production of legumes in the system of succession of sugarcane/legumes. The objective of this study was monitored the profile of agricultural production of temporary crops in the region of São Carlos, São Paulo, during 2004 to 2015. A meta-analysis was carried out with data from the Instituto Brasileiro de Geografia e Estatística. Monitored the production profile of sugarcane, peanuts, beans, cassava, corn, soybeans and tomatoes. Results showed that sugar cane and corn are the most expressive temporary crops. The increase in the agricultural area with sugarcane reduced the area of production of corn $(-0.85)$, soy $(-0.33)$, beans $(-0.74)$ and tomatoes $(-0.58)$. The importance of sugarcane production in the Brazilian scenario is evident for the production of sugar and ethanol. There is an inverse relationship between the sugarcane area and the areas with corn and soybeans, which are controlled by market conditions. In contrast, the succession using peanuts/sugarcane promotes a harmonious relationship between crops with increased peanut production and improved soil conditions, and an economically viable alternative in the reform of the cane field. Possibly, soybeans can also be included in this perspective in the coming years due to similar characteristics (also legumes) and the increase in demand for soy in the national and international market.
\end{abstract}

Keywords: Saccharum officinarum; Zea mays; Arachis hypogaea; Glycine max

\section{INTRODUÇÃ̃o}

A agricultura é palco de avanços científicos, socioculturais, tecnológicos e econômicos (NUNES, 2007; MAZOYER e ROUDART, 1993). Nas condições tropicais, a diversidade dos ecossistemas promove uma combinação de solos e condições climáticas que ocasionam o estabelecimento e produção agrícola de diferentes espécies (ALBUQUERQUE E SILVA, 2008; KLEIN, 1994; HARVEY et al. 2013). Dentre as espécies agrícolas cultivadas, o milho (Zea mays), trigo (Triticum) e arroz (Oryza sativa) são consideradas as espécies mais cultivadas no mundo (PAES, 2006). De acordo a FAO (2018) a produção global de trigo, milho e arroz crescerá 9,10 e $12 \%$ para suprir a alimentação humana até 2027

Focando na agricultura brasileira, o Brasil é considerado um dos maiores produtores de cana-de-açúcar (Saccharum officinarum), soja (Glycine max), milho, café (Coffea sp.), algodão (Gossypium hirsutum L.) e laranja (Citrus sinensis L. Osbeck). Esses produtos agrícolas são produzidos, principalmente nas regiões de São Paulo, Mato Grosso, Mato Grosso do Sul, Paraná, Bahia, e Minas Gerais, considerados como os grandes produtores brasileiros.

O estado de São Paulo destaca como o maior produtor de cana-de-açúcar e laranja in natura (CONAB, 2018). Na safra 2017/2018, São Paulo produziu 337,24 milhões de 
toneladas de cana-de-açúcar em uma área de 4.426,2 mil hectares (CONAB, 2018). Neste cenário, a região de São Carlos, localizada no interior do estado de São Paulo, atua como um importante "player" na produção agrícola (IBGE, 2017). Historicamente, a região de São Carlos surgiu no contexto da expansão da lavoura cafeeira no Brasil em solos com alta fertilidade natural. Na crise cafeeira de 1929, a agricultura perdeu força e deu espaço para a industrialização e fortalecimento da infraestrutura urbana (BRANDÃO, 2010).

A produção de cana-de-açúcar no Brasil foi intensificada após o estabelecimento do programa Proálcool, em 1975, com crescimento contínuo até o período entre 1998 e 2001, quando ocorreu uma queda na produção como reflexo da crise no setor. $\mathrm{O}$ crescimento acelerado foi retomado em 2003, com o lançamento e sucesso dos carros flexfuel no mercado brasileiro, com foco na produção do álcool hidratado (PAULILLO et al. 2007).

O aumento da produção de cana-deaçúcar no estado de São Paulo está mudando o perfil da agricultura no estado. Estudos realizados por Guilardi et al. (2015) demonstraram que entre 1988 e 2015 as áreas de cana-de-açúcar aumentaram de 11,6\% (13.221 ha) para $37 \%$ (41.805 ha); enquanto as áreas de pastagem diminuíram de $42 \%$ (47.647 ha) para $16 \%$ (18.683 ha). Em 2016, a região de São Carlos produziu $62.500 \mathrm{Mg}$ laranja e 2.890.000 Mg de cana-de-açúcar, representando uma diminuição na produção de laranja (60\%), e um aumento na produção de cana-de-açúcar (5\%) em relação a 2011. Ambas as produções de laranja e cana-deaçúcar, representam cerca de $0,32 \%$ e $1,90 \%$ no Produto Interno Bruto na região de São Carlos (IBGE, 2017).

Essa mudança no perfil da agricultura também influencia o perfil do produtor rural. De acordo com Vieira e Vieira Júnior (2006) e Nodari e Guerra (2003) a influência tecnológica também é um fator que está mudando o perfil do produtor rural nos últimos doze anos. Freitas (2013) destaca a assistência técnica, as empresas multinacionais e o nível tecnológico como fatores que estão influenciando a agricultura brasileira. A disseminação do nível tecnológico está relacionada a política tecnológica e o acesso dos produtores rurais às políticas públicas. Essas mudanças estão transformando a econômica e a produtividade agrícola brasileira (BUAINAIN et al. 2013).

O perfil da agricultura brasileira é baseado na agricultura familiar que contribuiu com mais de $50 \%$ da produção total de alimentos no Brasil (EMBRAPA, 2017). Na agricultura familiar, a família deve possuir até quatro módulos fiscais, de acordo a região, com a utilização predominantemente de mão 
de obra familiar, sendo a agricultura a principal fonte de renda (Lei $\mathrm{n}^{\circ}$ 11.326/2006; BRASIL, 2006). De acordo com a FAO (2016), 84\% do total dos estabelecimentos agropecuários brasileiros pertence a grupos familiares e representa uma média de 4,4 milhões de estabelecimentos localizados principalmente na Região Nordeste. A agricultura familiar é responsável pela maior parcela da produção agrícola local contribuindo para a produção de mandioca (87\%), feijão (70\%), milho (46\%), café (38\%), arroz (34\%), trigo (21\%), e leite (60\%) (FAO, 2016). Diante a importância da agricultura familiar, estudos que avaliem o impacto da mudança dos cenários agrícolas nas regiões produtoras de alimento no Brasil são necessários para entender o perfil da agricultura, e, portanto, justificando a realização deste estudo.

Com a hipótese que o aumento da área de cana-de-açúcar tem uma relação inversa com a produção de grãos na região de São Carlos. O objetivo deste estudo foi monitorar o perfil da produção agrícola das culturas temporárias na região de São Carlos, São Paulo, durante 2004 a 2015.

\section{MAterial e Métodos}

\subsection{Caracterização da área de estudo}

O estudo foi realizado na região de São Carlos, interior do estado de São Paulo. O município possuiu uma área de 1.136,907 $\mathrm{km}^{2}$, composta por uma população estimada de 246.088 habitantes.

O clima da região é classificado como Cfa (clima temperado húmido com verão quente), de acordo a classificação de Köppen e Geiger, com temperatura média de $19,7^{\circ} \mathrm{C}$, alta umidade relativa do ar, e verões mais quentes (KOTTEK, 2006). A econômica é composta pelos setores Agropecuário (1\%), Industrial (33\%), Prestação de Serviços (43 $\%)$, Administração e Serviços Públicos (9\%), e impostos (13\%) (IBGE, 2013).

\subsection{Monitoramento dos dados}

A coleta dos dados foi baseada em uma meta análise da produção agrícola temporária. Os dados representam a produção de lavouras temporárias na Região de São Carlos, entre os anos de 2004 e 2015, totalizando 12 anos. A coleta dos dados foi realizada no Censo agropecuário, desenvolvido pelo Instituto Brasileiro de Geografia e Estatística - IBGE (IBGE, 2004-2015). Monitorou o perfil da produção de cana-de-açúcar, amendoim (Arachis hypogaea), feijão (Phaseolus vulgaris), mandioca (Manihot esculenta), milho, soja e tomate (Solanum lycopersicum), levando em consideração os dados de produtividade e área de produção. Para a 
produção dos cerais (milho, soja e feijão) considerou peso em grãos, enquanto para a produção de amendoim considerou o peso em casca. Também monitorou a produção de cana-de-açúcar direcionada para álcool e açúcar.

\subsection{Análise estatística}

A variabilidade temporal foi usada para explicar os dados de área e produção agrícola, e a quantidade de cana-de-açúcar direcionada para a produção de álcool e açúcar. A variabilidade temporal foi dividida em dois períodos: período 1 (2004/08); e período $2(2009 / 15)$.

Análises de correlação foram utilizadas para explicar a dinâmica da produção de cana-de-açúcar destinada para a produção de álcool e açúcar. Uma adicional estudo de correlação explicou a influência da área agrícola de cana-de-açúcar com as áreas agrícolas com milho, soja, feijão e tomate.

\section{ReSUltados E DiscuSSÃo}

\subsection{Monitoramento anual da área e produção}

A cana-de-açúcar e o milho são as culturas temporárias com maiores expressividades na região de São Carlos/SP. Enquanto, a soja, amendoim, feijão, mandioca e tomate apresentam uma menor representatividade (Figura 1)

No monitoramento anual verificou o crescimento da área cultivada com cana-deaçúcar após 2008 passando de uma média de área cultivada de 23.590,8 (2004/08) para 35.292,1 (2009/15), representando um aumento de 33\% (Tabela 1; Figura 1). Esse aumento da área produzida promoveu o aumento da produção de cana-de-açúcar representada por um acréscimo de $26 \%$ (Figura 1). Esse impulso na produção de canade-açúcar em 2008 ocorreu devido as ações geopolíticas para produção de biocombustíveis derivado da cana-de-açúcar (KOHLHEPP, 2010; GUILARDI et al. 2015). Em 2008, o Brasil estabeleceu, pela primeira vez, a obrigatoriedade do percentual mínimo de $2 \%$ de biodiesel ao óleo diesel, antes desta data o biodiesel podia ser consumido, mas sem a existência da mistura (GOMIDE, 2011). Atualmente, a obrigatoriedade de biodiesel ao óleo diesel é até $15 \%$ de biodiesel ao óleo diesel vendido ao consumidor final (ANP, 2019). Além disso, em 2008, também ocorreu uma maior demanda por açúcar no mercado mundial o que incentivou a produção (CARVALHO JUNIOR e BONE, 2010). Melo e Sampaio (2016) descrevem que o preço e a demanda do açúcar influenciam a produção do setor sucroalcooleiro, 
considerando o preço do açúcar mais influente que preço do etanol.

Em nosso estudo ambas as produções de álcool e açúcar cresceram entre 2004 a 2015, com uma significativa correlação positiva entre ambos. Esse resultado indica um crescente aumento das produções de açúcar e álcool com uma proporção de $1.0 \mathrm{Mg}$ de cana-de-açúcar destinada para produzir álcool; por $0.9 \mathrm{Mg}$ de cana-de-açúcar destinada para produção de açúcar (Figura 2).
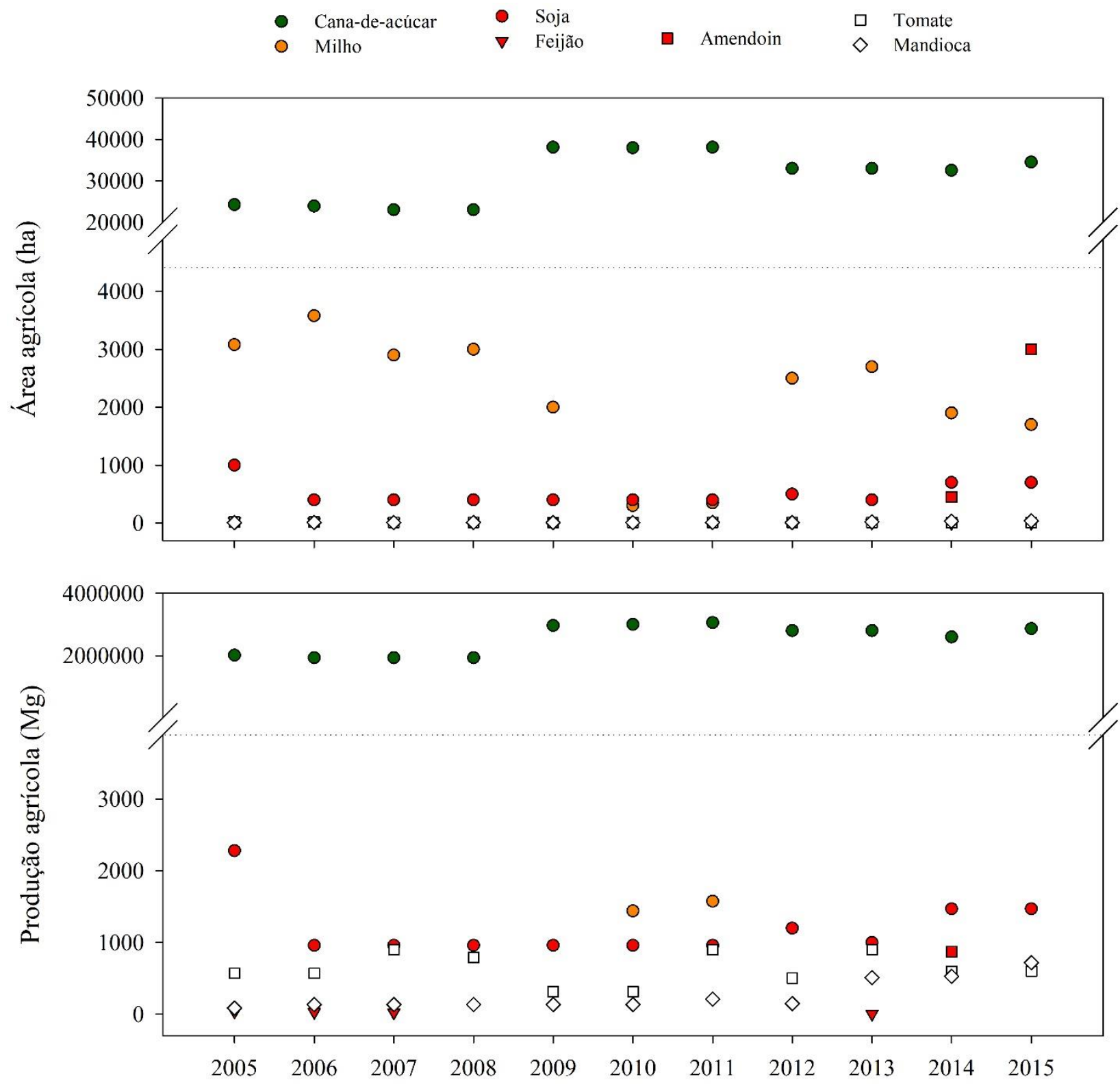

Anos

Figura 1. Área (hectare) e produção agrícola (Mg) de cana-de-açúcar, milho, soja, amendoim, feijão, mandioca, tomate na região de São Carlos, São Paulo, Brasil, durante 2005 a 2015. A produção de amendoim iniciou o monitoramento em 2014. 
Os dados demonstram que existe uma variação anual na produção de cana-de-açúcar com um desvio de $\pm 6.342,2$ ha na área cultivada, $\pm 488.385,4 \mathrm{Mg}$ na produção (Tabela 1; Figura 1). Essas variações ocorrem devido as condições climáticas, como descrito em trabalhos por Aude (1995), Gava et al. (2011); Gallucci et al. (2018). De acordo com Aude (1995) o perfilhamento da cana-deaçúcar que reflete na produtividade tem uma relação direta com os fatores climáticos e nutricionais. Gava et al. (2011) demonstrou que o adequado suplemento de água durante o desenvolvimento da cana-de-açúcar pode promover uma elevação de $24 \%$ na produtividade de colmos e de $23 \%$ na produtividade de açúcar. A necessidade da renovação a cada 5 anos (aproximadamente) também explica essa variação, pois a produção da cana-de-açúcar é reduzida com os anos, diminuindo o retorno financeiro ao longo do tempo (BARROS E MILAN, 2010).

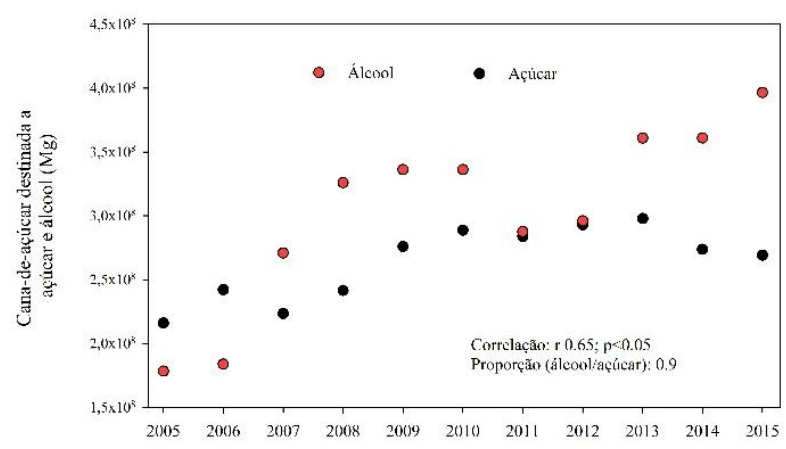

Figura 2. Produção de cana-de-açúcar destinada para a produção de álcool e açúcar entre 2004-2015.

\section{2 Áreas de produção agrícola}

O aumento na área agrícola com canade-açúcar impactou diretamente na produção de milho, soja, feijão e tomate representado por um r de correlação de $-0,85 ;-0,33 ;-0,74$; $-0,58(\mathrm{p}<0.05)$, respectivamente, Figura 3.

A alta correlação inversa entre as áreas de cana-de-açúcar e milho representaram uma diminuição da área agrícola com milho de $50 \%$ comparado o intervalo 2004/08 e 2009/15, Tabela 1. Possivelmente, a pressão do mercado pelo cultivo de cana-de-açúcar e a melhor facilidade no comercio e rentabilidade a partir de 2008 casou a diminuição da área cultivada de milho e, consequentemente, da produção do grão.

Interessantemente, entre 2010 e 2011, ocorreu um declínio acentuado na área de produção do milho (Figura 1). No entanto, a produção de milho teve um aumento. Possivelmente, esse resultado pode estar associado a utilização de novas variedades de milho transgênico, como o milho Bt (EMBRAPA, 2013). O cultivo de milharais com tecnologia Bt contribuíram para aumentar o lucro com uma média de $12 \%$ superior que a utilização de milho híbrido, e também aumentou a produtividade (OZELAME e ANDREATTA, 2013).

A menor correlação entre a cana-deaçúcar e a soja (r: -0,33) é explica pelo aumento da área agrícola a partir de 2014. A sucessão entre a cana-de-açúcar e soja vem 
sendo indicada com uma alternativa viável na reforma do canavial para a melhoria da qualidade do solo (SILVA e CASTRO, 2015). No entanto, em nosso estudo as áreas de produção com cana-de-açúcar e soja apresentam uma dinâmica competitiva entre as mesmas. Estudos realizados por Souza et al.
(2007) demonstraram que existe uma variação relativa das áreas cultivadas de soja e cana-deaçúcar no Brasil que é influenciado pela rentabilidade do mercado de ambas. Por exemplo, no período de 1994-2005, as áreas de soja substituíram as áreas com cana-deaçúcar devido a melhor condição de mercado.

Tabela 1. Área (hectare) e produção agrícola (Mg) de cana-de-açúcar, milho, soja, amendoim, feijão, mandioca, tomate na região de São Carlos, São Paulo, Brasil, durante 2005 a 2015.

\begin{tabular}{lccc}
\hline Cultivo & $\mathbf{2 0 0 4 / 0 8}$ & Área agrícola (ha) & Média \\
\hline Cana-de-açúcar & $23.590,8 \pm 558,2$ & $\mathbf{2 0 0 9 / 1 5}$ & $30.292,1 \pm 2.641,1$ \\
Milho & $3.228,0 \pm 327,6$ & $1.635,7 \pm 959,5$ & $2.299,2 \pm 1.101,6$ \\
Soja & $640,0 \pm 328,6$ & $500,0 \pm 141,4$ & $558,2 \pm 235,3$ \\
Feijão & $20,0 \pm 12,2$ & $0,7 \pm 1,9$ & $8,8 \pm 12,5$ \\
Amendoim & - & $492,9 \pm 1.118,2$ & $287,5 \pm 864,0$ \\
Mandioca & $8,4 \pm 1,3$ & $19,0 \pm 11,9$ & $14,6 \pm 10,4$ \\
Tomate & $12,8 \pm 2,2$ & $10,6 \pm 1,4$ & $11,5 \pm 2,0$ \\
\hline \multicolumn{4}{c}{ Produção agrícola (Mg) } \\
\hline Cana-de-açúcar & $2.117 .745,8 \pm 36.346,2$ & $2.869 .800,1 \pm 154.336,2$ & $2.485 .935,0 \pm 488.385,4$ \\
Milho & $13.370,2 \pm 5.796,3$ & $8.230,7 \pm 5.796,3$ & $10.686,3 \pm 5.316,7$ \\
Soja & $1.400,0 \pm 237,3$ & $1.145,7 \pm 237,3$ & $1.288,3 \pm 501,9$ \\
Feijão & $21,0 \pm 2,3$ & $0,9 \pm 2,3$ & $11,0 \pm 15,5$ \\
Amendoim & - & $1.196,4 \pm 2.798,7$ & $697,9 \pm 2.156,8$ \\
Mandioca & $126,8 \pm 240,3$ & $341,4 \pm 240,3$ & $251,3 \pm 209,9$ \\
Tomate & $594,2 \pm 241,9$ & $590,6 \pm 241,9$ & $615,3 \pm 215,1$ \\
\hline
\end{tabular}

Também existe uma relação entre as áreas com cana-de-açúcar e amendoim, no entanto, parece ser uma relação harmoniosa usando os dados de monitoramento da produção de amendoim 2014/15 (Figura 1). O cultivo do amendoim tem sido recomendado para melhorar a qualidade dos solos durante a reforma da cana-de-açúcar (CRUSCIOL e SORATTO, 2007; TASSO JÚNIOR, 2013; CARVALHO et al. 2004). Ambos o amendoim e a soja são leguminosas que tem a DOI: 10.24021/raac.v18i1.5218 capacidade de adicionar nitrogênio da atmosfera no solo devido a fixação biológica, denominada de adubação verde (FerrazAlmeida, 2020). Estudos realizados por Bolonhezi et al. (2007) mostraram que o índice de rendimento de grãos do amendoim aumenta de 6,5 a 9\% em sistemas com sucessão cana-de-açúcar/amendoim. Além disso, a presença da palha da cana construiu para aumentar o conteúdo de água no solo e o aporte de carbono. Em contrapartida, a canaVol. 18, N. 01 (2021) 
de-açúcar é beneficiada devido ao aumento de nitrogênio no solo com a fixação biológica promovida pelo amendoim, e que também melhora as condições do solo (LAZARINI e CRUSCIOL, 2000; BOLONHEZI et al. 2005). Possivelmente, nos próximos anos a sucessão da cana-de-açúcar/amendoim pode promover um aumento da área de produção do amendoim. Atualmente, o Estado de São Paulo é responsável por cerca de $90 \%$ de toda a produção de amendoim no Brasil, cujo destino é a exportação (80 \%) e consumo interno (20 \%). Desde 2009, a produção nacional de amendoim cresce em uma taxa anual média de $13 \%$ no Brasil, enquanto em São Paulo esse crescimento anual médio é de $16 \%$ (CONAB, 2018).

A área cultivada com o tomate também foi afetada com o aumento da área agrícola com cana-de-açúcar com um $\mathrm{r}$ de -0.58 (Figura 3), e representando uma redução de 2.2 ha e 3,6 toneladas entre 2004/08 e 2009/15
(Tabela 1). O tomate é uma das hortaliças mais consumidas no mundo e uma das culturas mais importantes no Brasil, onde sua produção pode ser direcionada para processamento industrial ou in natura (CEPEA, 2013). No cenário de tomates para processamento industrial, o Brasil é o $5^{\circ}$ maior produtor mundial e o estado que mais se destaca é Goiás com uma produção de 1.440.961 Mg (33 \% da produção nacional), CONAB (2019). Possivelmente, o cultivo de tomate na região de São Carlos deve estar associado a agricultura familiar, e essa diminuição é explicada pelo arrendamento de áreas agrícolas na região para a produção de cana-de-açúcar. 


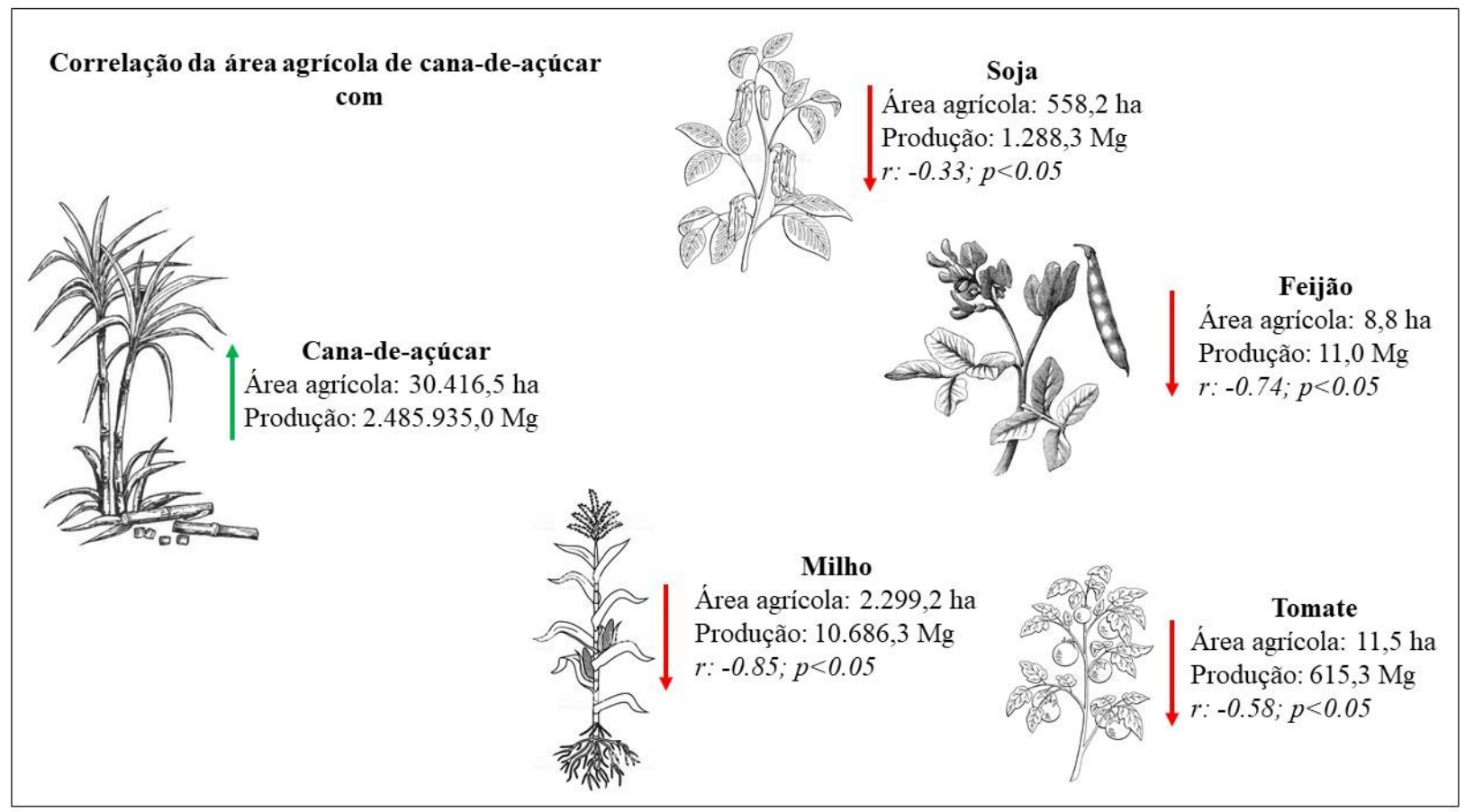

Figura 3. Correlação da área agrícola de cana-de-açúcar como a área agrícola com milho, soja, feijão e tomate na região de São Carlos, São Paulo, Brasil, durante 2005 a 2015. Valor da correlação destacada em itálico.

\section{CONCLUSÕES}

Nos últimos 12 anos, a produção de cana-de-açúcar apresentou uma relação inversa com a produção de milho, tomate e soja. Em contrapartida, a sucessão amendoim/cana-de-açúcar promoveu uma relação harmoniosa com a produção de canade-açúcar e amendoim. A sucessão com leguminosas é uma alternativa economicamente viável na reforma do canavial. Possivelmente, a soja também pode ser inserida nesta perspectiva nos próximos anos devido as características similares (também uma leguminosa) e aumento da demanda por soja no mercado nacional e internacional.

\section{Agradecimentos}

Ao MBA da USP/ESALQ (Universidade de São Paulo/Escola Superior de Agricultura "Luiz de Queiroz") pelo inventivo e apoio.

\section{REFERÊNCIAS}

ANP - Agência Nacional do Petróleo, Gás Natural e Biocombustíveis. Óleo diesel passa a conter mínimo de $11 \%$ de biodiesel a partir de $1^{\circ}$ de setembro (2019). Disponível: http://www.anp.gov.br/noticias/5298-oleodiesel-passa-a-conter-minimo-de-11-debiodiesel-a-partir-de-1-de-setembro.

Acessado: Junho, 2020

ALBUQUERQUE, A. C. S.; SILVA, A. G. da2008. Agricultura tropical: quatro 
décadas de inovações tecnológicas, institucionais e políticas. EMBRAPA, Brasília, 2008. 1340 p.

AUDE MIS. Estádios de desenvolvimento da cana-de-açúcar e suas relações com a produtividade. Ciência Rural, Santa Maria, v. 23, n. 2, p. 241-248, 1993.

BARROS FF, MILAN M. Qualidade operacional do plantio de cana-de-açúcar. Bragantia, Campinas, v. 69, n. 1, p. 221-229, 2010

BOLONHEZI D, GODOY IJ, SANTOS RC. Manejo da cultura do amendoim. In: SANTOS, R.C. dos (Ed.). O agronegócio do amendoim no Brasil. Campina Grande: Embrapa Algodão, 2005. 193-244 p.

BOLONHEZI D, MUTTON MÂ, MARTINS, MELLO AL. Sistemas conservacionistas de manejo do solo para amendoim cultivado em sucessão à cana crua. Pesquisa agropecuária brasileira, Brasília, v. 42, n. 7, p. 939-947, 2007.

BRANDÃO AAL. A carta do ex-escravo Felício \& História de São Carlos (SP). São Carlos: Fundação Pró-Memória de São Carlos, 2010. 15p.

Brasil. 2006. Estabelece as diretrizes para a formulação da Política Nacional da Agricultura Familiar e Empreendimentos Familiares Rurais. Lei No 11.326, DE 24 DE JULHO DE 2006. Disponível: <http://www.planalto.gov.br/ccivil_03/_Ato2 004-2006/2006/Lei/L11326.htm>. Acessado: Junho, 2020
BUAINAIN AM, ALVES E, SILVEIRA JM, NAVARRO Z. Sete teses sobre o mundo rural brasileiro. Revista de Política Agrícola, Brasília, v. 2, p. 105-121, 2013.

CARVALHO MAC, ATHAYDE MLF, SORATTO RP, ALVES MC, SÁ ME. Adubação verde e sistemas de manejo do solo na produtividade do algodoeiro. Pesquisa Agropecuária Brasileira, Brasília, v. 39, p. 1205-1211, 2004.

CARVALHO JUNIOR LJ, BONE RB. Demanda brasileira de cana de açúcar, açúcar e etanol revisitada. XXX Encontro Nacional de Engenharia De Produção: Maturidade e desafios da Engenharia de Produção: competitividade das empresas, condições de trabalho, meio ambiente. Anais. São Carlos, SP, Brasil, 12 a15 de outubro de 2010.

CEPEA - Centro de Estudos Avançados em Economia Aplicada. Tomate. Hortifruti Brasil, Piracicaba, v. 130, n.1, p. 31-34, 2013.

CONAB - Companhia Nacional de Abastecimento. 2018. Levantamento da produção agrícola de cana-de-açúcar. Disponível: <https://www.conab.gov.br/infoagro/safras/cana/boletim-da-safra-de-canade-

acucar/item/download/17727_4e54c5103a0a b4a15529e35307c79b2e>. Acessado: Junho, 2020

CONAB - Companhia Nacional de Abastecimento. 2017. Levantamento da safra brasileira. Disponível em <http://www.conab.gov.br>. Acesso em: nov. 2019 
CONAB - Companhia Nacional de Abastecimento. 2017. Tomate: Análise dos Indicadores da Produção e Comercialização no Mercado Mundial, Brasileiro e Catarinense. Disponível em < https://www.conab.gov.br/institucional/publi cacoes/compendio-de-estudos-daconab/item/download/29586_4fe6dd2c9c6d1 fa5e1cbc5f82061717d>. Acesso em: nov. 2019

CRUSCIOL CAC, SORATTO RP. Nutrição e produtividade do amendoim em sucessão ao cultivo de plantas de cobertura no sistema plantio direto. Pesquisa Agropecuária brasileira, Brasília, v. 42, n. 11, p. 15531560. 2007.

EMBRAPA - Empresa Brasileira de Pesquisa Agropecuária. A real contribuição da agricultura familiar no Brasil. Disponível: < https://www.embrapa.br/agropensa/busca-denoticias/-/noticia/27405640/a-realcontribuicao-da-agricultura-familiar-nobrasil>. Acessado: Junho, 2020

GAVA GJC, SILVA MA, SILVA RC, JERONIMO EM, CRUZ JCS, KÖLLN OT. Produtividade de três cultivares de cana-deaçúcar sob manejos de sequeiro e irrigado por gotejamento. Revista Brasileira de Engenharia Agrícola e Ambiental, Campina Grande, v. 15, n. 3, p. 250-255, 2011.

GALLUCCI AD, NATERA M, MOREIRA LA, et al. Nitrogen-enriched vinasse as a means of supplying nitrogen to sugarcane fields: testing the effectiveness of $\mathrm{n}$ source and application rate. Sugar Tech, Heidelberg, Springer Nature, p. 1-9, 2018.
FAO. 2016. Family Farming Knowledge Platform.

Disponível:

http://www.fao.org/family-

farming/detail/en/c/454156/. Acessado:

Junho, 2020

FAO. 2018. Disponível em: <http://www.fao.org/3/i9166e/i9166e_Chapt er3_Cereals.pdf>. Acessado: Junho, 2020

FERRAZ-ALMEIDA, R.; DA SILVA, N.; WENDLING, B. How Does $\mathrm{N}$ Mineral Fertilizer Influence the Crop Residue N Credit?. Nitrogen, v. 1, p. 99-110, 2020.

GUILARDI V., RONQUIM CR, GONÇALVES JMC. Dinâmica as áreas de cana-de-açúcar e pastagem nos municípios de Barretos e São Carlos entre os anos de 1988 e 2015. Anais. IX Worshop Agroenergia, Ribeirão Preto, 2015.

GOMIDE R.B. 2010. Políticas governamentais para o $\mathbf{s}$ biocombustíveis. Capt. 11. Disponível em: $<$ https://www.biodieselbr.com/pdf/cap11.pdf >. Acessado: Junho, 2020

IBGE - Instituto Brasileiro de Geografia e Estatística. 2017. Produção Agrícola Municipal 2009-2016. Disponível em < https://www.ibge.gov.br $>$.

IBGE - Instituto Brasileiro de Geografia e Estatística. Levantamento da safra brasileira 2004-2017. Disponível em <https://www.ibge.gov.br>. Acesso em: nov. 2019. 
IBGE - Instituto Brasileiro de Geografia e Estatística. 2013. Produto Interno Bruto dos Municípios. Disponível em: $<$ https://www.ibge.gov.br/estatisticasnovoportal/economicas/contasnacionais/9088-produto-interno-bruto-dosmunicipios.html? $=\& \mathrm{t}=\mathrm{o}-\mathrm{que}-\mathrm{e}>$. Acesso em: nov. 2019.

HARVEY et al. Climate-Smart Landscapes: Opportunities and Challenges for Integrating Adaptation and Mitigation in Tropical Agriculture. A journal of the society for conservatuib biology. v.7, n.2, p. 77-90, 2013

KOHLHEPP G. 2010. Análise da situação da produção de etanol e biodiesel no Brasil. Disponível de $<$ http://www.scielo.br/scielo.php?script=sci_ arttext\&pid=S0103$40142010000100017 \& \operatorname{lng}=$ en $\& n r m=i s o>$. Acesso em: nov. 2019.

KLEIN RG. Chasse, cueillette et agriculture en Afrique. Paris: Bordas, col. "Les berceaux de I'humanité', 1994.

KOTTEK M, GRIESER J, BECK C, RUDOLF B, RUBEL F. World Map of the Köppen-Geiger climate classification updated. Meteorologische Zeitschrift, Switzerland, v. 15, n. 3, p. 259-263, 2006

LAZARINI E, CRUSCIOL CAC. Produtividade do amendoim da seca em função do sistema de produção e da época de semeadura. Revista de Agricultura, Piracicaba, v. 75, p. 287-301, 2000
MAZOYER M, ROUDART L. História das agriculturas no mundo: do neolítico à crise contemporânea. São Paulo, Editora UNESP, 1993

MELO AS, SAMPAIO YSB. Uma Nota Sobre o Impacto do Preço do Açúcar, do Etanol e da Gasolina na Produção do Setor Sucroalcooleiro. Revista Brasileira de Economia, Rio Janeiro, v. 70, n. 1, p. 61-69., 2016.

NUNES SP. O desenvolvimento da agricultura brasileira e mundial e a ideia de Desenvolvimento Rural. DESER (Departamento de Estudos SócioEconômicos Rurais) - Boletim Eletrônico. $\mathrm{N}^{\circ}$ 157, 2007.

OZELAME O, ANDREATTA T. Avaliação de desempenho técnico e econômico: um estudo comparativo entre o milho híbrido e o milho Bt. Custos e @gronegócio, Recife,v. 9. n. 1, p. 2010-232, 2013.

PAES MCD. Aspectos físicos, químicos e tecnológicos do grão de milho. Sete Lagoas: Embrapa Milho e Sorgo, 2006

PAULILLO LF, VIAN CEF, SHIKIDA PFA, MELLO FT. Álcool combustível e biodiesel no Brasil: quo vadis?. Revista de Economia e Sociologia Rural, Brasília, v. 45, n. 3, p. 531-565, 2007

SILVA AA, CASTRO SS. Indicadores macro e micromorfológicos da qualidade física de um latossolo vermelho cultivado com canade-açúcar. Mercator, Fortaleza, v.14, n. 3, p. 169-185, 2015. 
SOUZA GS, ALVES E, GOMES EG, GAZZOLA R, Marra. Substituição de culturas: Uma abordagem empírica envolvendo cana-de-açúcar, soja, carne bovina e milho. Revista Política agrícola. Brasília, v. 16, n. 2, p. 5-13, 2007.

TASSO JÚNIOR, L.C. Cultura da soja, milho e amendoim sob diferentes sistemas de manejo do solo em área com palha residual de colheita mecanizada de cana crua. 2003. 154p. Dissertação (Mestrado) Universidade Estadual Paulista, Jaboticabal.

VIEIRA AC, VIEIRA JÚNIOR PA. Debates atuais sobre a segurança dos alimentos transgênicos e os direitos dos consumidores. Direito do consumidor, Porto Alegre, v. 5, n. 1, p. 25-47, 2006. 